\title{
Commissioning of the Superconducting ECR ion source VENUS at $18 \mathrm{GHz}$
}

D. Leitner, S.R. Abbott, R.D. Dwinell, M.A. Leitner, C. Taylor, C.M. Lyneis,

\author{
Nuclear Science Division, Lawrence Berkeley National Laboratory, Berkeley, California 94720
}

During the last year, the VENUS ECR ion source was commissioned at $18 \mathrm{GHz}$ and preparations for $28 \mathrm{GHz}$ operation are now underway. During the commissioning phase with $18 \mathrm{GHz}$, tests with various gases and metals have been performed with up to $2000 \mathrm{~W}$ RF power. The ion source performance is very promising $[1,2]$.

VENUS (Versatile ECR ion source for NUclear Science) is a next generation superconducting ECR ion source, designed to produce high current, high charge state ions for the 88-Inch Cyclotron at the Lawrence Berkeley National Laboratory. VENUS also serves as the prototype ion source for the RIA (Rare Isotope Accelerator) front end.

The goal of the VENUS ECR ion source project as the RIA R\&D injector is the production of $240 \mathrm{e} \mu \mathrm{A}$ of $\mathrm{U}^{30+}$, a high current medium charge state beam. On the other hand, as an injector ion source for the 88-Inch Cyclotron the design objective is the production of $5 \mathrm{e} \mu \mathrm{A}$ of $\mathrm{U}^{48+}$, a low current, very high charge state beam. To meet these ambitious goals, VENUS has been designed for optimum operation at $28 \mathrm{GHz}$.

This frequency choice has several design consequences. To achieve the required magnetic confinement, superconducting magnets have to be used. The size of the superconducting magnet structure implies a relatively large plasma volume. Consequently, high power microwave coupling becomes necessary to achieve sufficient plasma heating power densities. The $28 \mathrm{GHz}$ power supply has been delivered in April 2004.

\section{COMMISSIONING RESULTS AT 18 GHZ}

In 2002 and 2003 initial commissioning at $18 \mathrm{GHz}$ was carried out. During this commissioning period, a number of improvements were made to the cryostat system, the $18 \mathrm{GHz}$ microwave system, and the magnet power supply control system $[1,2]$.

Novel heat exchangers for the two cryocoolers were designed, and they enable VENUS to operate in closed loop operation without the addition of liquid helium. Recently a third cryocooler was added in preparation for the $28 \mathrm{GHz}$ operation. The third cryocooler adds $1.3 \mathrm{~W}$ of additional cooling power to the cryostat system and enables to run the magnet at the design temperature of $4.2 \mathrm{~K}$. It also provides ample cooling power compensating the expected increased heat load at $28 \mathrm{GHz}$ operation due to the x-ray load produced by hot plasma electrons colliding with the plasma chamber.

VENUS is now operational at the full capacity of the $2 \mathrm{~kW}, 18 \mathrm{GHz}$ klystron. The operation experience has been excellent. In table 1 a few exemplary ion beam intensities from VENUS are presented. The performance of VENUS exceeds the performance of the LBNL AECR-U in all areas.

Table 1: Ion beam intensities extracted from VENUS

\begin{tabular}{|l|l|l|l|l|l|l|}
\hline $\mathrm{O}^{6+}$ & $\mathrm{O}^{7+}$ & $\mathrm{Xe}^{20+}$ & $\mathrm{Xe}^{27+}$ & $\mathrm{Bi}^{25+}$ & $\mathrm{Bi}^{29+}$ & $\mathrm{Bi}^{41+}$ \\
\hline 1100 & 324 & 164 & 84 & 164 & 115 & 11 \\
\hline
\end{tabular}

In August 2003 a high temperature oven has been installed in VENUS. A prototype of this oven has been developed and successfully tested previously with the existing LBNL ECR ion sources for temperatures up to 2000 degree C. Bismuth was selected as the first metal ion beam to be produced with VENUS. Figures $1 \mathrm{a}$ and $1 \mathrm{~b}$ show a Bi spectrum optimized for high and low charge states.
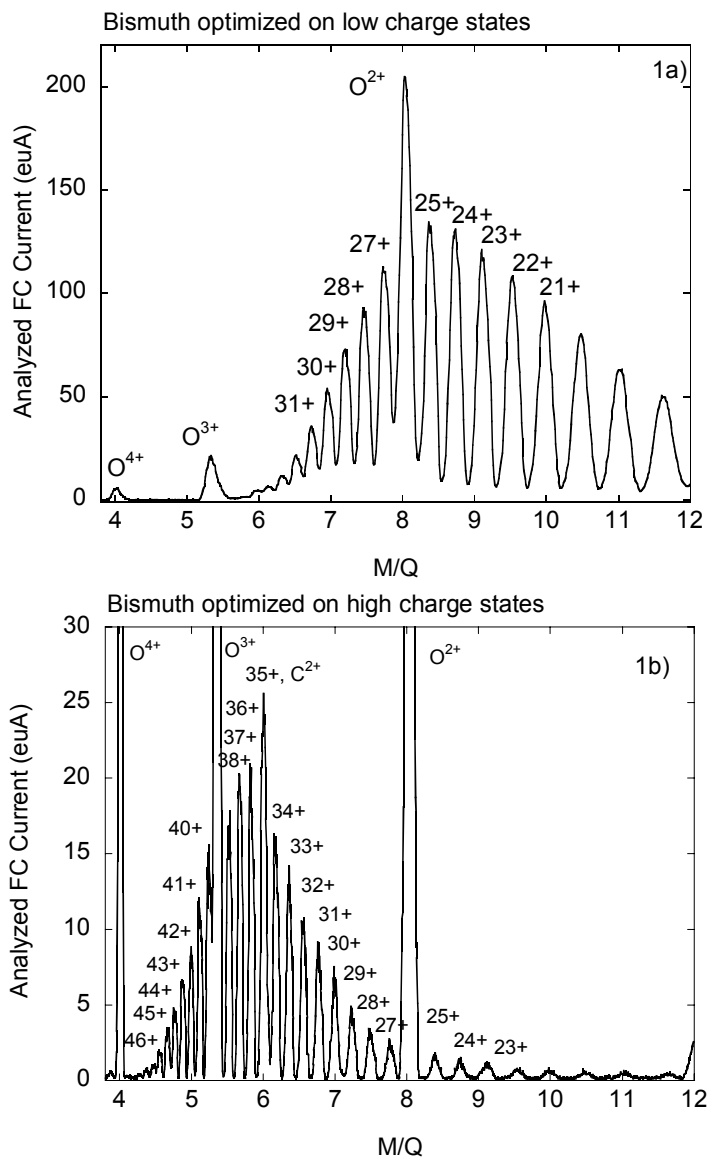

Fig.1 Analyzed Bi current for an ion source tune optimized for low (1a) and high (1b) charge states. Note the different current scales in the spectra.

\section{REFERENCES}

[1] D. Leitner, S. R. Abbott, R. D. Dwinell, M. Leitner, C. Taylor, and C. M. Lyneis, APS Proceedings of the Particle Accelerator Conference (PAC'03), Portland, Or, 2003).

[2] C.M. Lyneis, D. Leitner, S.R. Abbott, R.D. Dwinell, M. Leitner, C.S. Silver, C. Taylor, RSI75, in print, 2004 